\title{
Benign intracranial hypertension after ciprofloxacin administration
}

\author{
A P Winrow, G Supramaniam
}

\begin{abstract}
Many drugs, including antibiotics, have been implicated in the aetiology of benign intracranial hypertension. We report the development of benign intracranial hypertension after the use of ciprofloxacin in a teenager with cystic fibrosis.
\end{abstract}

Benign intracranial hypertension has been linked with several different aetiological factors. Many of these implicate drugs, particularly antibiotics, ${ }^{1}$ in the development of benign intracranial hypertension. The girl with cystic fibrosis presented in this case report had been treated with an antipseudomonal antibiotic not previously associated with benign intracranial hypertension.

\section{Case report}

A 14 year old girl with cystic fibrosis presented with a $\mathbf{1 0}$ day history of headache and vomiting. Her headache resolved after two days, although her nausea and vomiting persisted. She also complained of intermittent diplopia. Despite the diagnosis of cystic fibrosis, her general health and respiratory function were well maintained. Recent recurrent chest symptoms had been initially treated with cefaclor then changed to ciprofloxacin after 10 days because of a poor response to the former antibiotic. Her current medication also included pancreatin, vitamin supplements (Ketovite, Paines and Byrne), carbohydrate supplements (Maxijul, Scientific Hospital Supplies), with ciprofloxacin $250 \mathrm{mg}$ twice daily.

Examination showed a well nourished girl continuing her growth pattern with her height on the 50th centile and weight on the 10th centile. A left VIth cranial nerve palsy and bilateral papilloedema were noted. Visual acuity was reduced to $6 / 9$ on the left. Perimetry did not demonstrate a visual field deficit. There was no evidence of meningism, ataxia, or cerebellar dysfunction. She was normotensive. Respiratory examination showed a mildly hyperexpanded thorax with scattered inspiratory crepitations. There was evidence of early puberty. She was transferred to the neurosurgical department of the Hospital for Sick Children, London.

Plasma electrolyte estimations were normal. Cranial computed tomography showed ventricular compression without cerebral oedema or space occupying lesion. Lumbar puncture showed normal cerebrospinal fluid at a raised pressure of $41 \mathrm{~cm}$ cerebrospinal fluid (normal range 7-18). A diagnosis of benign intracranial hypertension was made. At that stage, erythromycin was substituted for ciprofloxacin, on the assumption that ciprofloxacin was the only recent change in treatment and therefore the likely cause of the problem. Two further lumbar punctures were performed which showed persistently raised cerebrospinal fluid pressures each treated with removal of 10 to 25 ml of fluid. There was subsequent symptomatic improvement with resolution of the cranial nerve palsy but with residual papilloedema. Further lumbar puncture three weeks later showed a pressure of $23 \mathrm{~cm}$ cerebrospinal fluid, which was treated with removal of a further 25 $\mathrm{ml}$ of fluid. There was further resolution of the papilloedema and the nerve palsy did not recur.

\section{Discussion}

Benign intracranial hypertension was initially associated with impaired cerebrospinal fluid absorption resulting from lateral sinus thrombosis after acute otitis media, hence the term 'post otitic hydrocephalus'. ${ }^{2}$ It has also been associated with 'catch up' growth in cystic fibrosis after a period of relative malnutrition. ${ }^{3}$ In this case, however, the growth pattern had been well maintained. Various drugs have been implicated including tetracyclines ${ }^{4}$ and nitrofurantoin. ${ }^{5}$ We are unaware of any previous reports linking the administration of the 4quinolone antibiotic ciprofloxacin and benign intracranial hypertension. Since this report was submitted, we are aware of a recent additional case occurring in an adult in the USA during ciprofloxacin treatment for sinusitis (personal communication, Bayer UK Ltd). Such an association has been documented with the use of nalidixic acid ${ }^{6}$ which is the parent compound for the quinolone series of antibiotics. The above association is important because of the increasing popularity of the quinolone group of antibiotics and their use as oral antipseudomonal agents particularly in those with cystic fibrosis. Ciprofloxacin is being advertised for use in general practice for many infections. It has been extensively used in cystic fibrosis for pseudomonas infection. With increasing use there is a possibility of developing a resistant organism and benign intracranial hypertension should be considered if headache is a new symptom.

We would like to thank Mr DN Grant and his neurosurgical colleagues at the Hospital for Sick Children, Great Ormond Street, London, for their help in the management of this patient.

1 Askmark H, Lundberg PO, Olsson S. Drug-related headache. Headache 1989;29:441-4.

2 Symonds CP. Post-otitic hydrocephalus. Brain 1931;54:55 
3 Bray RF, Herbst JJ. Pseudotumour cerebri as a sign of 'catch-up' growth in cystic fibrosis. Am $\mathcal{f}$ Dis Child 1973;126:78-9.

4 Minutello JS, Dimaynga RG, Carter J. Pseudotumour cerebin; a rare adverse reaction to tetracycline therapy. cerebin; a rare adverse reaction
$\mathcal{J}$ Periodontol $1988 ; 59: 848-51$.
5 Korzets A, Rathaus M, Chen B, Bernheim J. Pseudotumour cerebi and nitrofurantoin. Drug Intell Clin Pharm 1988;22: 345 .

6 Dekurto E, Manzani C, Zecca G. Convulsions with cerebrospinal fluid hypertension during treatment with naladixic acid. Minerva Pediatr 1986;38:794-5.

\title{
$5 \alpha-$ Reductase deficiency without hypospadias
}

\author{
W K Ng, N F Taylor, I A Hughes, J Taylor, P G Ransley, D B Grant
}

\begin{abstract}
A boy aged 4 with penoscrotal hypospadias and his brother aged 12 with micropenis had typical changes of homozygous $5 \alpha$-reductase deficiency. After three injections of chorionic gonadotrophin there was a trivial rise in plasma dihydrotestosterone with a normal increase in plasma testosterone. Urine steroid chromatography showed abnormally high $5 \beta$ : $5 \alpha$ ratios and $5 \alpha$-reductase activity was appreciably reduced in genital skin fibroblasts. The results indicate that $5 \alpha$-reductase deficiency is not invariably associated with genital ambiguity.
\end{abstract}

$5 \alpha$-Reductase deficiency is a well recognised but uncommon cause of male pseudohermaphroditism. ${ }^{1}$ Published cases have been characterised by pronounced underdevelopment of the male external genitalia with microphallus and incomplete fusion of the labioscrotal folds. ${ }^{2}$ Many of the reported cases have been initially raised as girls because of the female appearance of the genitalia at birth but cases with more pronounced virilisation have been described. ${ }^{3}$

All previously reported males with $5 \alpha$ reductase deficiency have had some degree of genital ambiguity with hypospadias. We describe two brothers with the conditicn, one of whom had micropenis without hypospadias while the other had penoscrotal hypospadias.

Hospital for

Sick Children,

Great Ormond Street, London WCIN 3JH

W $\mathrm{K} \mathrm{Ng}$

J Taylor

P G Ransley

D B Grant

Department of Clinical Biochemistry,

King's College

School of Medicine

and Dentistry,

London

N F Taylor

Department of

Paediatrics,

University of Cambridge

School of Clinical

Medicine, Cambridge

I A Hughes

Correspondence to:

Dr Grant (no reprints

Dr Grant (noilable).
a

Accepted 9 June 1990

(Arch DisChild 1990;65:1166-7)

\section{Patients and methods}

The propositus and his older brother had consanguineous parents who come from Pakistan. At birth, the younger boy was found to have abnormal external genitalia with a small phallus and hypospadias. When he was 4 years old he was referred for evaluation of his genital anomaly. At that time he was noted to have a small penis (stretched length $1.9 \mathrm{~cm}$ ) with noticeable chordee and penoscrotal hypospadias. The scrotum was normal but only the right testis was palpable.

Initial investigations showed a 46XY karyotype. A sinogram showed a normal bladder; the proximal urethra had a male configuration with a prominent vermontarum and well developed utricle.

After initial evaluation he was given one injection of depot testosterone $(50 \mathrm{mg})$ to improve the size of his penis and hypospadias repair was carried out. A skin biopsy specimen for fibroblast culture was taken at that time.

The older brother was noted to have a small penis at birth. He was seen at the age of 12 years, at the same time as his brother. His external genitalia were normal, apart from the size of his penis, which had a stretched length of $4 \mathrm{~cm}$. There was no chordee and the urethra opened at the tip of the penis. Both testes were in the scrotum and were $6 \mathrm{ml}$ in volume.

Investigation showed a 46XY karyotype. After initial investigation a skin biopsy specimen was obtained from the scrotum.

Plasma testosterone and dihydrotestosterone concentrations were measured by radioimmunoassay before and after three daily injections of 1000 units of human chorionic gonadotrophin. Urine steroid concentrations were measured by gas chromatography as previously described, ${ }^{4}$ and the results expressed in terms of $5 \beta: \alpha$ ratios for different steroid metabolites.

Fibroblasts were established in culture from genital skin to determine $5 \alpha$-reduct.se activity and androgen receptor concentrations. The activity of $5 \alpha$-reductase was measured after incubation of fibroblasts with serum free medium containing $2 \mathrm{nM}$ of tritiated testosterone. The medium was extracted with ethyl acetate and analysed by single step thin layer chromatography. Areas corresponding to dihydrotestosterone, $5 \alpha$-androstanedione, androsterone, epiandrosterone, and the $5 \alpha$-androstanediols were cut out and counted for tritium. Enzyme activity was expressed in pmol/ mg protein/hour of $5 \alpha$-reduced products. The concentration of androgen receptors, receptor binding affinity, presence or absence of heat labile receptors, and effect of preincubation

Table 1 Results of endocrine investigations

\begin{tabular}{|c|c|c|}
\hline & Propositus & Brother \\
\hline $\begin{array}{l}\text { Before human chorionic gonadotrophin: } \\
\text { Testosterone (nmol/l) } \\
\text { Dihydrotestosterone (nmol/l) } \\
\text { Testosterone:dihydrotestosterone ratio }\end{array}$ & $\begin{array}{l}0 \cdot 4 \\
0 \cdot 1 \\
4 \cdot 0\end{array}$ & $\begin{array}{l}3 \cdot 1 \\
0 \cdot 2 \\
1 \cdot 5\end{array}$ \\
\hline $\begin{array}{l}\text { After human chorionic gonadotrophin: } \\
\text { Testosterone (nmol/l) } \\
\text { Dihydrotestosterone (nmol/l) } \\
\text { Testosterone:dihydrotestosterone ratio }\end{array}$ & $\begin{array}{c}4 \cdot 2 \\
0 \cdot 1 \\
42 \cdot 0^{*}\end{array}$ & $\begin{array}{c}21 \cdot 9 \\
0 \cdot 3 \\
73 \cdot 0^{*}\end{array}$ \\
\hline $\begin{array}{l}\text { Urinary } 5 \beta: 5 \alpha \text { ratios: } \\
\text { THF:alloTHF† } \\
\text { Aetiocholanolone:androsteroneł } \\
\text { THB:alloTHB }\end{array}$ & $\begin{array}{r}24 \cdot 7 \\
0.6 \\
0.8\end{array}$ & $\begin{array}{r}14 \cdot 3 \\
4 \cdot 7 \\
4 \cdot 3\end{array}$ \\
\hline
\end{tabular}

${ }^{*}$ Normal range in prepubertal boys:2-25. ${ }^{1}$

Mean (SD) results in normal subjects: $\dagger 1 \cdot 1(1 \cdot 7), \neq 0 \cdot 8(1 \cdot 7)$, $\$ 0 \cdot 7(\mathbf{1} \cdot 6)$. 